\title{
Modeling Dust Emission of HL Tau Disk Based on Planet-Disk Interactions
}

\author{
Sheng Jin ${ }^{1,2}$, Shengtai $\mathrm{Li}^{2}$, Andrea Isella ${ }^{3}$, Hui Li ${ }^{2}$, Jianghui Ji ${ }^{1}$
}

\begin{abstract}
We use extensive global two-dimensional hydrodynamic disk gas+dust simulations with embedded planets, coupled with three dimensional radiative transfer calculations, to model the dust ring and gap structures in the HL Tau protoplanetary disk observed with the Atacama Large Millimeter/Submillimeter Array (ALMA). We include the selfgravity of disk gas and dust components and make reasonable choices of disk parameters, assuming an already settled dust distribution and no planet migration. We can obtain quite adequate fits to the observed dust emission using three planets with masses 0.35 , 0.17 , and $0.26 M_{\text {Jup }}$ at $13.1,33.0$, and $68.6 \mathrm{AU}$, respectively. Implications for the planet formation as well as the limitations of this scenario are discussed.
\end{abstract}

Subject headings: protoplanetary disks — planet-disk interactions - techniques: interferometric — submillimeter: planetary systems

\section{Introduction}

ALMA observations of HL Tau, a young ( $\leq 1-2$ Myr ) star in Taurus, revealed bright and dark rings in the millimeter-wave continuum emission (ALMA Partnership et al. 2015). One interesting question is whether these structures are created by the embedded planets, hence yielding potentially important clues on planet formation in such disks.

Other mechanisms besides the effects of planets have been suggested, including zonal flows (Ruge et al. 2013), Rossby wave instability (Lovelace et al. 1999; Li et al. 2000, 2005; Regály et al. 2012; Pinilla et al. 2012), rapid pebble growth around condensation fronts (Zhang et al. 2015), and dust dynamics (Gonzalez et al. 2015). Some observational features suggest however that the pattern of rings shown in HL Tau disk may be produced by planets. First, the spectral index derived from the ALMA images suggests that the dark rings are optical thin whereas the bright regions are optically thick, therefore the dark rings are real gaps in the dust distribution (ALMA Partnership et al. 2015). Second, the increase of the eccentricity of the rings at large orbital

\footnotetext{
${ }^{1}$ Key Laboratory of Planetary Sciences, Purple Mountain Observatory, Chinese Academy of Sciences, Nanjing 210008, China

${ }^{2}$ Theoretical Division, Los Alamos National Laboratory, Los Alamos, NM 87545

${ }^{3}$ Rice University, Houston, TX
} 
radii (ALMA Partnership et al. 2015) is consistent with the fact that the orbital eccentricities of exoplanets increase with orbital radii (Butler et al. 2006; Shen \& Turner 2008; Zhang et al. 2014). Third, the dust size constrained by the polarized emission is around $150 \mu m$ (Kataoka et al. 2015), which means the structure of multiple rings should also exist in the gas disk because dust at this size should be well coupled with gas. Pinte et al. (2015) conclude that the depletion of dust at each of the deepest gaps is up to $40 M_{\oplus}$, which is close to the point of runaway gas accretion.

Models of disks that couple the dynamics of gas, dust and planets are crucial to interpret the observed patterns in the HL Tau system (e.g., Dong et al. 2014; Zhu et al. 2015; Dipierro et al. 2015). Various models suggest that the disk mass of HL Tau is $\sim 0.03-0.14 M_{\odot}$ (Robitaille et al. 2007; Guilloteau et al. 2011; Kwon et al. 2011, 2015), and the estimated stellar mass of HL Tau is 0.55 to $1.3 M_{\odot}$ (Sargent \& Beckwith 1991; Close et al. 1997; White \& Hillenbrand 2004; ALMA Partnership et al. 2015). The high disk mass might affect the disk stability and the resonance locations of potential planets (Tamayo et al. 2015). A recent study by Dipierro et al. (2015) used a three-dimensional gas+dust two-fluid SPH code that includes embedded planets. Though they were able to reproduce the pattern of bright and dark rings, they adopted a disk mass of only $0.0002 M_{\odot}$ within $120 \mathrm{AU}$ and under predicted by about a factor 20 the observed millimeter flux density.

In this paper, we perform quantitative fitting to the ALMA observations of HL Tau in terms of its millimeter flux density and spatial variations. We address the question whether the observed features of HL Tau by ALMA can be produced by planet-disk interactions. We describe our approach and model set-up in Section 2, summarize our main results in Section 3, and discuss their implications in Section 4.

\section{Models}

The model adopted to analyze HL Tau observations makes use of the LA-COMPASS code (Li et al. 2005, 2009; Fu et al. 2014) to calculate the planet-disk interaction, and of radiative transfer code $R A D M C-3 D^{1}$ to calculate the disk temperature and emission at $1 \mathrm{~mm}$. Our aim is not to obtain a perfect fit to the ALMA observation of HL Tau but instead to investigate in a quantitative way whether the planet-disk interactions might explain the observed features.

\subsection{Disk gas+dust+planet model}

We initialize our hydrodynamic simulation using the results from Kwon et al. (2011). The disk extends from 2.4 AU to $156 \mathrm{AU}$, and is simulated using a polar grid containing 3072 and 768 cells

\footnotetext{
${ }^{1}$ http://www.ita.uni-heidelberg.de/ dullemond/software/radmc-3d/
} 
in the radial and azimuthal direction, respectively. We adopt a disk surface density described by

$$
\Sigma_{g}(r)=\Sigma_{0}\left(\frac{r}{r_{0}}\right)^{0.23} \exp \left[-\left(r / r_{c}\right)^{2.23}\right]
$$

where $r_{c}=79 \mathrm{AU}$ is a characteristic radius (e.g., Andrews et al. 2009) and $r_{0}=10 \mathrm{AU}$. The mass of the star is $M_{*}=0.55 M_{\odot}$. Although Kwon et al. (2011) finds a disk mass of $0.135 M_{\odot}$, we find that the disk with this mass quickly becomes gravitationally unstable under small perturbations. Hence we reduce the disk mass to be $M_{\text {disk }} \approx 7.35 \times 10^{-2} M_{\odot}$, which leads to $\Sigma_{0}=4.83 \times$ $10^{-4}\left[M_{*} /(10 \mathrm{AU})^{2}\right] \approx 23.61 \mathrm{~g} / \mathrm{cm}^{2}$. The whole disk is assumed to have a constant Shakura-Sunyaev viscosity parameter $\alpha=10^{-3}$. The locally isothermal sound speed is chosen as:

$$
\left(\frac{c_{s}}{v_{\phi}}\right)(r)=0.05\left(\frac{r}{r_{0}}\right)^{0.285} \text {. }
$$

We assume that the initial dust surface density $\Sigma_{d}(r)$ follows $\Sigma_{g}(r)$, with an initial dust-to-gas ratio of $1 \%$. We have performed gas+dust two-fluid simulations using different dust particle sizes ranging from $\mu \mathrm{m}$ to millimeter, though only one dust size is used in a single hydro simulation. Such gas+dust distributions are used to calculate the dust temperature as well as the dust emissions which are ultimately compared with ALMA observations. For comparison with the ALMA images, we have used the 0.15 millimeter size dust particles from gas+dust two-fluid simulations (see below for dust models). The initial Stokes number of 0.15 millimeter size dust particles is $<0.01$ within $\sim 100$ AU. For such small Stokes number, we adopt short friction time approximation (Johansen \& Klahr 2005) to circumvent problems with small time steps.

The disk self-gravity and indirect acceleration term from the central star are always included. An extended disk that includes inner disk $r \in[0.01,0.24]$ and outer disk $r \in[15.6,156]$ is used in disk self-gravity calculations. We emphasize that the effects of both disk self-gravity and indirect acceleration term are important due to the relatively massive disk in HL Tau. In fact, simulations without including such terms could give erroneous results. A fixed boundary condition is used for gas at both inner and outer disk boundaries. For dust, we adopt an inflow (outflow) boundary condition at the inner (outer) boundary.

ALMA Partnership et al. (2015) identify seven pairs of dark and bright rings in the $1.0 \mathrm{~mm}$ image of the HL Tau disk, and among them four prominent dark rings could be considered as gaps, i.e., the dark rings D1, D2, and the adjoining D5 and D6. Based on a large body of previous literature on gap opening and formation as functions of disk gas temperature, planet mass, disk viscosity (e.g., Crida, Morbidelli, \& Masset 2006; Li et al. 2009), we have performed tens of disk + planet hydro simulations in order to determine the likely planet mass values that could roughly match the multiple gap widths and depths as shown in the ALMA observations. Based on these simulations, we adopt a nominal model (run0) which has a set of planet mass parameters as $M_{p} \approx 0.35,0.17$, and $0.26 M_{\mathrm{Jup}}$ at $13.1,33.0$, and $68.6 \mathrm{AU}$, respectively. These three planets are fixed at their orbital locations, and planetary radial migration is turned off. 
To explore how our results could vary with some key parameters, we have carried out many additional runs. In Table 1, we list a few simulations where we reduce the masses for all three planets by half (run1), disable the disk self-gravity (run2), and reduce the disk viscosity (run3). Other changes such as the disk surface density and mass are not presented here. Most of the results presented in this paper use the snapshot at 4000 orbits $(\sim 170,556$ years at $10 \mathrm{AU})$ to generate simulated observations, except for run1 where the snapshot at 3400 orbits is used.

\subsection{Dust model, radiative transfer model and image generation}

The dust opacities adopted in this work were calculated as in Isella, Carpenter \& Sargent (2009) by assuming that dust grains are compact spheres made of astronomical silicates (Weingartner \& Draine 2001), organic carbonates (Zubko et al. 1996), and water ice, with fractional abundances as in Pollack et al. (1994). Single grain opacities were averaged on a grain size distribution to obtain the mean opacity used in the radiative transfer model. We did that by adopting a typical MNR power-law size distribution (Mathis, Rumpl, \& Nordsieck 1977), $n(a) \propto a^{-3.5}$, between a minimum grain size of $5 \times 10^{-4} \mathrm{~mm}$ and a maximum grain size of $10 \mathrm{~mm}$. The resulting dust opacity at the wavelength of $1 \mathrm{~mm}$ is $4.6 \mathrm{~cm}^{2} \mathrm{~g}^{-1}$, and it is dominated by dust grains with sizes between 0.1 and $0.2 \mathrm{~mm}$. Based on this assumption, in our hydrodynamic models we adopt dust grains with a size of $0.15 \mathrm{~mm}$ as best tracers of the dust emission at $1 \mathrm{~mm}$.

To calculate synthetic maps of the disk continuum emission, we first need to calculate the dust temperature throughout the disk. This is controlled by micron-size dust particles that are well coupled with gas. We adopt a dust-to-gas ratio of 0.01 and use the gas surface density from simulations to interpolate the micron-size dust density distribution on a 3D spherical grid along with

a scale height profile of, $h_{\mu \mathrm{m} \text {-dust }}(r)=1.0 \mathrm{AU} \times(r / 20 \mathrm{AU})^{1.25}$. We set up a thermal Monte Carlo run to calculate the dust temperature using the radiative transfer code $R A D M C$-3D. The calculated dust temperature profile shows the typical two-layer vertical structure of passive irradiated circumstellar disks (Dullemond et al. 2002). In the surface layer, the temperature decreases from $\sim 280 \mathrm{~K}$ at 5 $\mathrm{AU}$ to $\sim 75 \mathrm{~K}$ at $100 \mathrm{AU}$. In the midplane, the temperature decreases from $\sim 110 \mathrm{~K}$ at $5 \mathrm{AU}$ to $\sim$ $20 \mathrm{~K}$ at $100 \mathrm{AU}$. Next, using the obtained dust temperature, we calculate the millimeter thermal emission based on the density and opacity of our dust disk. We convert the surface density of 0.15

Table 1: Simulation Parameters

\begin{tabular}{lcccc}
\hline Model & DSG & $M_{\mathrm{p}}\left(M_{\text {Jup }}\right)$ & $\alpha$ & orbit no. \\
\hline run0 & $\mathrm{Y}$ & $0.35,0.17,0.26$ & $1 \mathrm{e}-3$ & 4000 \\
run1 & $\mathrm{Y}$ & $0.17,0.09,0.13$ & $1 \mathrm{e}-3$ & 3400 \\
run2 & $\mathrm{N}$ & $0.35,0.17,0.26$ & $1 \mathrm{e}-3$ & 4000 \\
run3 & $\mathrm{Y}$ & $0.35,0.17,0.26$ & $2 \mathrm{e}-4$ & 4000 \\
\hline
\end{tabular}


millimeter size dust from two-fluid simulations to a 3D spherical density profile using a scale height of, $h_{\text {mm-dust }}(r)=f_{\text {sett }} \times h_{\mu \mathrm{m} \text {-dust }}(r)$, where $f_{\text {sett }}=0.1$ is a parameter that accounts for the settling of 0.15 millimeter size dust towards the mid-plane. This leads to a scale height of $\sim 0.75 \mathrm{AU}$ at 100 $\mathrm{AU}$ for relatively large dust particles, which is consistent with the findings by Pinte et al. (2015). Using our gaseous disk parameters, the estimated settling time of $0.15 \mathrm{~mm}$ dust is within $10^{5}$ years at the scale height of gas disk, thus it is a reasonable choice of dust scale height, considering the age of the HL Tau disk. We then generate the synthetic images at the millimeter wavelength using the ray tracing method of $R A D M C$ - $3 D$. As a last step, we "observe" our models and compare them with the ALMA observations in the Fourier domain, following the procedure described in (Isella, Carpenter \& Sargent 2009).

\section{Results}

\subsection{The nominal model}

Figure 1 shows the surface density distributions of gas and 0.15 millimeter size dust in our nominal model. The gas and dust disks exhibit similar morphology, showing three gaps and three higher density rings/bands of gas created by the disk-planets interactions, along with visible spiral arm features. The rings/bands in the dust distribution show asymmetries associated with the spiral density features produced by the planets, especially in the inner and middle rings. The dust to gas surface density ratio (initially at 0.01) shows the evolution of dust+gas distributions while dust is subject to gas drag and is concentrated at high pressure region, causing significantly enhancements at the locations of rings. Dust filtration effects are expected in the gaps because the larger size dust particles are more concentrated in the rings. The deficit of dust at large radii $>120 \mathrm{AU}$ is due to the fact that dust particles drift radially inward and there is no supply of dust particles through the outer boundary.

Since HL Tau system is relatively young, the viscous timescale at disk radii $>10$ AU is longer than the age of the disk. So, the disk is not expected to be in quasi-steady state. When monitoring the evolution of the ring and gap structures over time, we indeed find that the accretion rate in simulations is not steady and the depths of the gaps are still evolving (say between 4000 and 7000 orbits), though understandably the first gap is approaching a steady state faster.

Figure 2 shows the ALMA observation of HL Tau, the synthetic observations of our nominal model, and a residual map obtained by subtracting the model from the observations in the Fourier domain. Overall, our model reproduces the observations quite well. (Good agreement is obtained between our model and ALMA Band 6 and 7 observations, and we are only showing the Band 7 image due to its higher spatial resolution.) What is most remarkable is that the flux density level between the model and observations can be matched so closely, given the fact that we have used a generic smooth gaseous disk with a uniform initial dust to gas ratio throughout such a disk. The simulated observation of our model can explain pretty well the three major gaps at $\sim 13,33$, and 

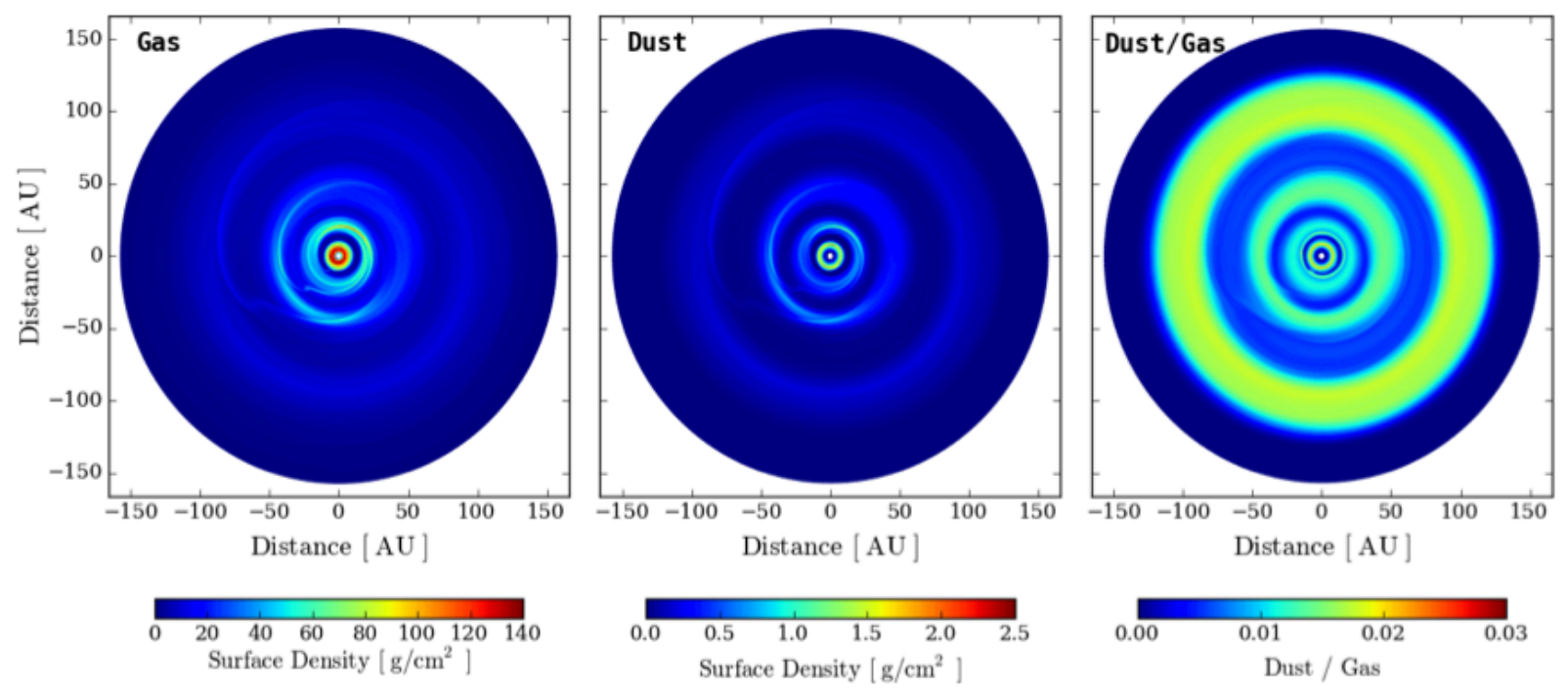

Fig. 1. - Surface density of the gas (left) and of $0.15 \mathrm{~mm}$ size dust grains (center) after 4000 orbits for our nominal model that has three planets with masses of $0.35,0.17$, and $0.26 M_{\mathrm{Jup}}$ at 13.1 , 33.0, and 68.6 AU, respectively. The right panel shows the dust-to-gas ratio.

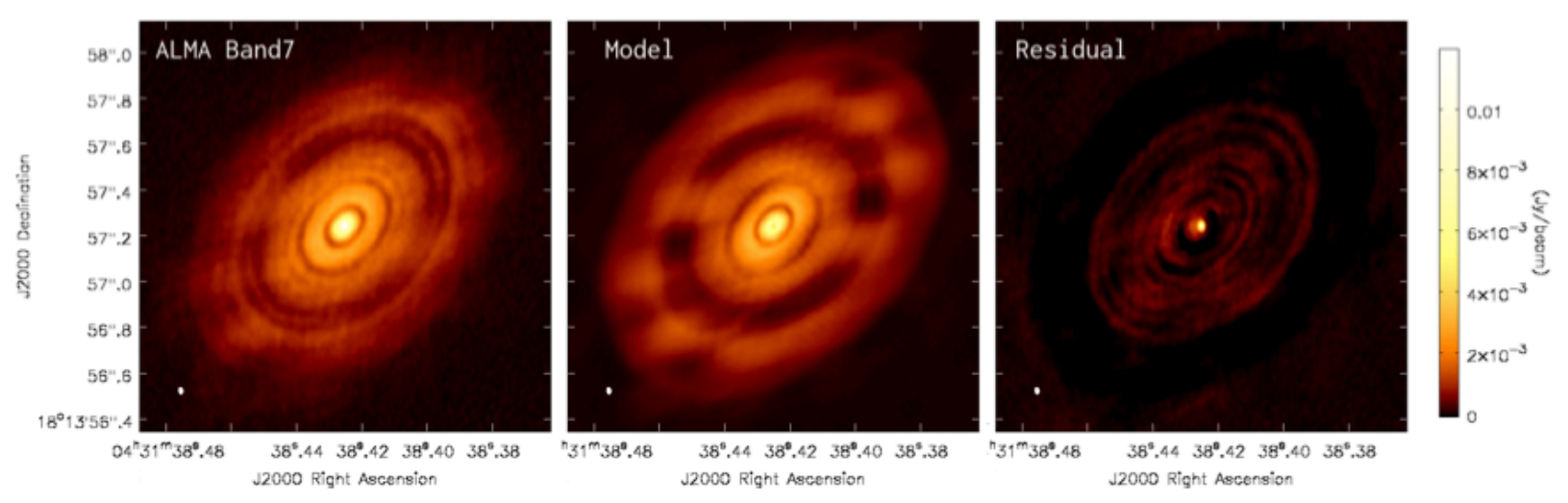

Fig. 2.- Comparison between the ALMA map of the HL Tau disk (left) and the simulated observation of our nominal model (middle). The right panel shows the residual, i.e. the difference between the observation and our model. The white point in the lower left corner shows the synthesized beam. 
$68 \mathrm{AU}$, but it does not reproduce the detailed structures of multiple rings at larger radii due to our choice of using only one planet at $68 \mathrm{AU}$. The large difference at the center region shown in the residual is due to our choice of $2.4 \mathrm{AU}$ as the inner disk boundary. Interestingly, the spiral arms and the asymmetry in the rings that are visible in the gas and dust distributions (cf Figure 1) are mostly smeared out in the simulated observation due to the high optical depth of the continuum emission at $850 \mu \mathrm{m}$.

One of the key findings from the ALMA observations is that rings and gaps are somewhat eccentric. To quantify this feature in our model image, by using a face-on configuration, we find the local maxima (for the rings) or minima (for the gaps) in the azimuthal direction and fit the locations of these points with ellipses. We obtain the eccentricities of gaps as $0.246,0.274$ and 0.277 , respectively, whereas the eccentricities of rings as $0.191,0.079$ and 0.157 , respectively. This reproduces the trend seen in the actual ALMA observations, though quantitative comparisons are somewhat difficult. This reproduces the trend seen in the actual ALMA observations. Because we have calculated the eccentricities using our simulations which have much higher spatial resolutions than ALMA observations, simulations can yield more pronounced non-axisymmetric features on smaller scales, this makes more quantitative comparisons with observations difficult.

The left panel of Figure 3 shows the radial profile of the intensity along the major axis of the disk corresponding to a position angle of $138^{\circ}$. The flux densities of our model match the observation well, especially in the region around the first and second ring+gap. At the third ring and beyond, the relative difference between our model and the observation increases. Again, it is worthwhile emphasizing that the relative heights among different regions were all obtained self-consistently through two-fluid gas+dust simulations along with the radiative transfer calculations.

The right panel of Figure 3 shows the optical depth $\tau$ and the spectral index $\alpha$ of the dust emission calculated between $0.87 \mathrm{~mm}$ and $1.3 \mathrm{~mm}$ along the disk major axis. The dark rings are (partially) optically thin while the rest of the disk is mostly optically thick. For $\tau \ll 1$, $\alpha$ depends on the slope of the dust opacity $\beta(\alpha \sim 2+\beta)$. Vice versa, for $\tau \gtrsim 1, \alpha$ approaches 2 . The radial profile of $\alpha$ predicted by our model is also in remarkable agreement with the observations (see Figure 3 of ALMA Partnership et al. 2015).

Finally, note that since we used only one dust size in our simulations, the spectral index variation in our model is only determined by the properties of the dust disk (such as optical depth). In real observations, the radial variation of spectral index might also depend on the dust properties (such as size evolution) through the disk (Guilloteau et al. 2011; Testi et al. 2014).

\subsection{Influence of parameters}

In Figure 4 we compare the other three models with the ALMA Band 7 image. Similar with Figure 3, we draw cross-cuts of the simulated observations of run1, 2, and 3 along the major axis

of the disk and compare the obtained flux density profiles with the observation. Run1, where the 

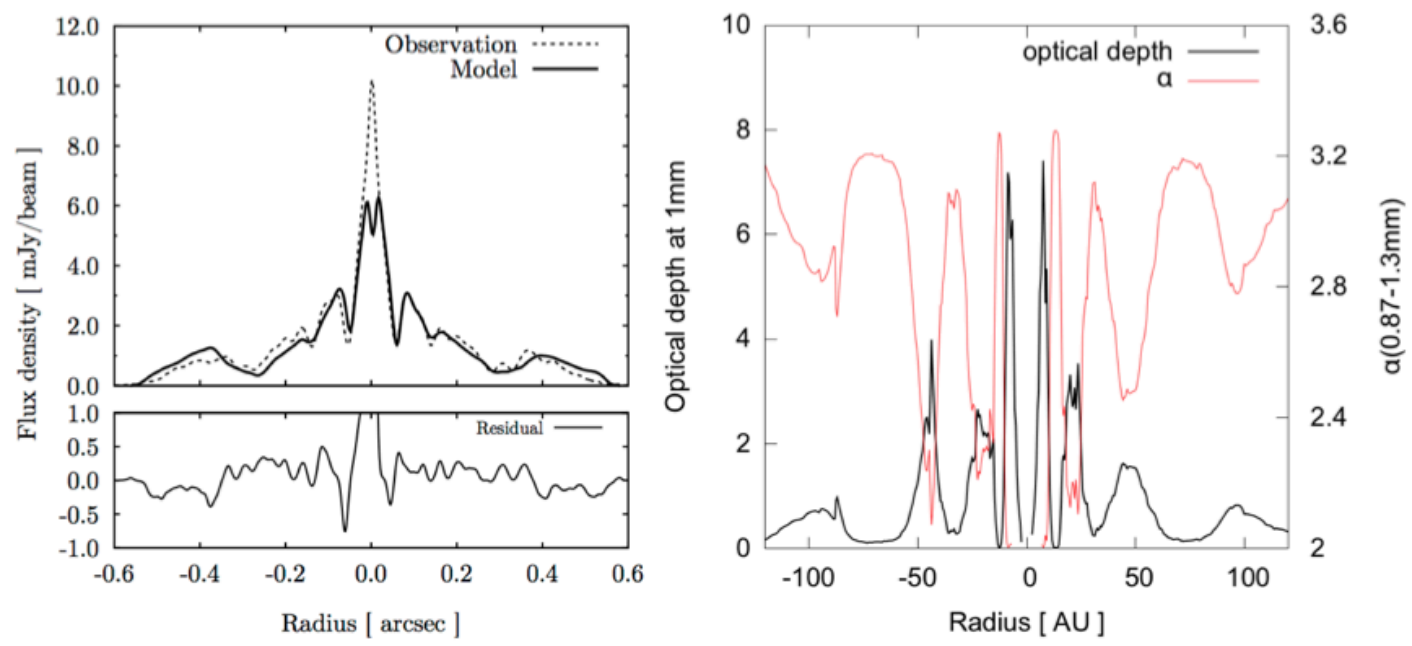

Fig. 3.- Left: Comparison of the flux densities of our model and the ALMA Band 7 observation using a line cut along the major axis of the disk. The bottom panel shows the residual. Right: Optical depth at 1 millimeter (dark line) and millimeter spectral index $\alpha$ of the model emission using a line cut along the major axis of the disk. The dark ring regions are mostly optical thin whereas other parts of the disk are optically thick.
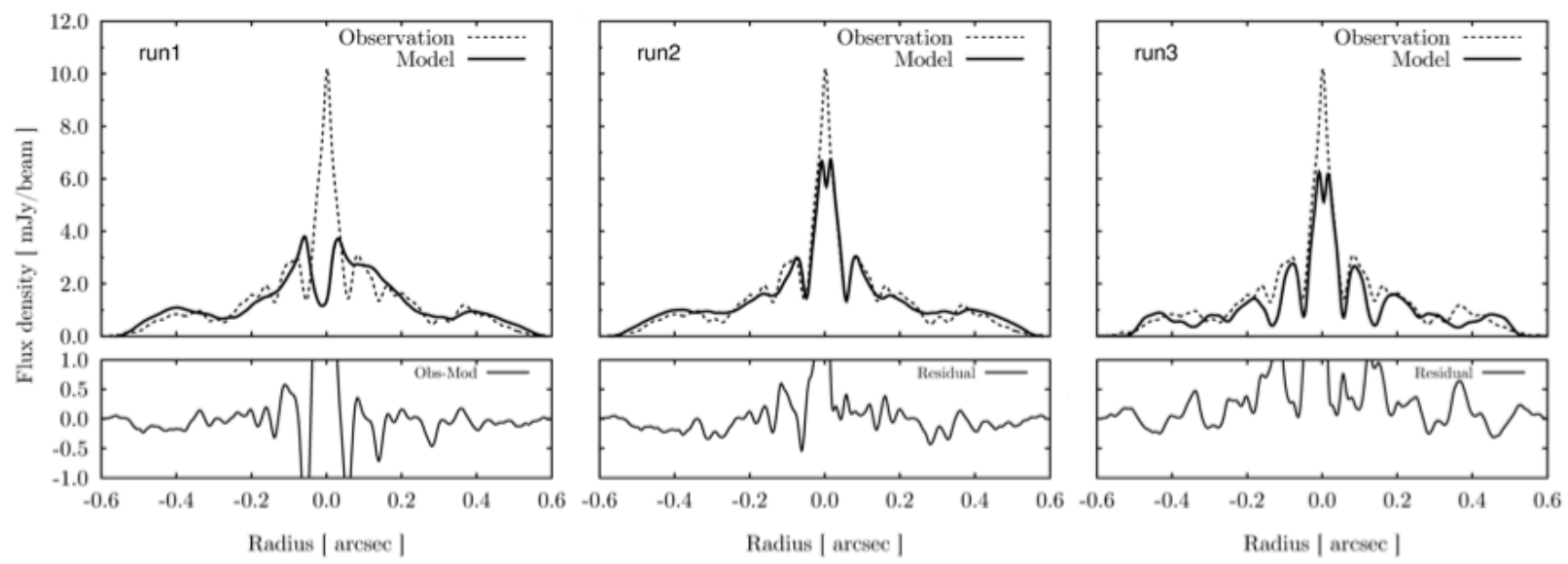

Fig. 4.- Comparison of the flux densities along the disk major axis for model run1, 2, and 3, and the ALMA observation. 
planet masses are half of the nominal model, fails to produce the second gap and the depth of the first gap is too shallow compared to the observation. This is expected since less massive planets can not open deep gaps. Run2, where the disk self-gravity is turned off, fails to produce the third gap. This is because, as the third planet gradually creates a gap, the gravitational force of the disk interior of the planet will try to pull the outer disk inward, further promoting the formation of a partial gap. Without such disk self-gravity, the gap is much shallower. In run3, where the disk viscosity is reduced by a factor of 5 , the gaps are too deep because the spiral shocks produced by planets in low viscosity disks are more effective in clearing the regions surrounding the planets.

Note that the results shown in Figure 4 do not mean that we could not find a suitable fitting when parameters are different from our nominal model. On the contrary, it is likely that a right combination of planet masses, disk viscosity, temperature, mass, as well as the disk evolution time could be found to produce a reasonable fit to the current ALMA observation. The parameter studies in this subsection are meant to help us to gauge how sensitive the final outcome is to various parameters.

\section{Summary and Discussion}

We have performed 2D hydrodynamic simulations of disk gas+dust evolution including disk self-gravity and embedded planets to model the HL Tau disk. The simulation results are then coupled with 3D radiative transfer calculations to be compared with the observation of Partnership et al. (2015). By comparing synthetic emission maps and observations in the Fourier domain, we have tested whether the observed multiple rings and gaps could be generated by the diskplanet interactions. Through a large number of simulations, we find that the observed features can indeed be mostly reproduced by planet-disk interactions. Without any fine-tuning, we find that disk model containing three planets with masses of $\sim 0.35,0.17,0.26 M_{\mathrm{Jup}}$, located at 13.1,33.0, and 68.6 AU, respectively, is able to reproduce the the radial profile of the flux density, as well as, its spectral index. The rings and gaps from the model are also eccentric, consistent with the ALMA observations. Furthermore, our fitted model parameters favor a relatively massive disk and an initial dust to gas ratio about 0.01 . The effects of self-gravity are essential in reproducing the profiles for gaps and rings.

There are, however, several outstanding issues that deserve further studies. First, the three planets in our model are assumed to stay on fixed radii. When allowed to migrate, they eventually move out of the current locations. Although the observed gaps seem to be in resonance, it is difficult to understand why planets will be in such resonances, given the fact that their radial migration speeds tend to be fast in a high-mass disk environment (cf. Zhang et al. 2014). Second, we find that the parameters we adopted for the disk and planets are barely able to keep the disk stable

and with low eccentricity (we will present additional results in a future publication). The disk self-gravity, as well as the indirect acceleration term, are playing an important role in such disks. In general, contrary to the quasi-axisymmetry of the observed HL Tau image, the disk and planet 
orbits are expected to be more eccentric for such disk and planet masses. Third, previous studies have suggested that HL Tau could be an FU Ori system in quiescence (Lin et al. 1994), which means that mass must be accumulating in the disk. In addition, the presence of jet/outflows suggests that the angular momentum transport processes in this disk are quite complicated. This non-steady situation, coupled with uncertainties in the lifetime of planets, makes it difficult to constrain the planet mass more precisely.

Nonetheless, the high-resolution ALMA observations of HL Tau system have enabled detailed modeling of this system, and we expect observations at longer wavelengths of this system from JVLA will further constrain the models. Using the same model in this paper but with $1.5 \mathrm{~mm}$ dust grain size (the dust that dominates the centimeter emission), we find that the rings we saw at $1 \mathrm{~mm}$ become much narrower at the $1 \mathrm{~cm}$ wavelength because the effect of dust concentration at high pressure regions is more prominent for larger grains. We also find that these three bright rings are still optical thick at $1 \mathrm{~cm}$ wavelength. Future observations of the morphology of HL Tau system at longer wavelengths can provide additional constraints for understanding dust dynamics and planet formation.

This paper makes use of the ALMA data. The Joint ALMA Observatory is operated by ESO, AUI/NRAO and NAOJ. Support by LANL's LDRD, UC-Fee and IGPPS programs are gratefully acknowledged. J.H. and S.J. appreciate the support by National Natural Science Foundation of China (grants no. 11273068, 11473073, 11503092), the Strategic Priority Research Program-The Emergence of Cosmological Structures of the Chinese Academy of Sciences (grant no. XDB09000000), the innovative and interdisciplinary program by CAS (grant no. KJZD-EW-Z001), the Natural Science Foundation of Jiangsu Province (grant no. BK20141509) and the Foundation of Minor Planets of Purple Mountain Observatory. A.I. acknowledge support from NSF (grant no. 1535809) and NASA (grant no. NNX15AB06G). All computations are carried out using LANL's Institutional Computing resources. We thank C. Dullemond for making RADMC-3D available and for useful

discussions. Discussions with L. Perez are gratefully acknowledged as well. We thank the referee for comments that helped to improve the manuscript.

\section{REFERENCES}

Partnership, A., Brogan, C. L., Perez, L. M., et al. 2015, ApJ, 808, L3

Andrews, S. M. et al. 2009, ApJ, 700, 1502

Butler, R. P., Wright, J. T., Marcy, G. W., et al. 2006, ApJ, 646, 505

Close, L. M., Roddier, F., Hora, J. L., et al. 1997, ApJ, 489, 210

Crida, A., Morbidelli, A., \& Masset, F. 2006, ICARUS, 181, 587 
Dipierro, G., Price, D., Laibe, G., et al. 2015, arXiv:1507.06719

Dong, R., Zhu, Z., \& Whitney, B. 2014, arXiv:1411.6063

Dullemond, C. P., van Zadelhoff, G. J., \& Natta, A. 2002, A\&A, 389, 464

Fu, W., Li, H, Lubow, S., Li, S., \& Liang, E. 2014, ApJL, 795, L39

Gonzalez, J.-F., et al., 2015, MNRAS, in press (arXiv:1509.00691)

Guilloteau, S., Dutrey, A., Piétu, V., \& Boehler, Y. 2011, A\&A, 529, A105

Johansen, A., \& Klahr, H. 2005, ApJ, 634, 1353

Isella, A., Carpenter, J. M., \& Sargent, A. I. 2009, ApJ, 701, 260

Kataoka, A., Muto, T., Momose, M., Tsukagoshi, T., \& Dullemond, C. P 2015, arXiv:1507.08902

Kwon, W., Looney, L. W., \& Mundy, L. G. 2011, ApJ, 741, 3

Kwon, W., Looney, L. W., Mundy, L. G., \& Welch, W. J. 2015, ApJ, 808, 102

Li, H., Finn, J.M., Lovelace, R.V.E., \& Colgate, S.A. 2000, ApJ, 533, 1023

Li, H., et al. 2005, ApJ, 624, 1003

Li, H., Lubow, S. H., Li, S., \& Lin, D. N. C. 2009, ApJL, 690, L52

Lin, D.N.C., et al. 1994, ApJ, 435, 821

Lovelace, R.V.E., Li, H., Colgate, S.A., \& Nelson, A.F. 1999, ApJ, 513, 805

Mathis, J. S., Rumpl, W. \& Nordsieck, K. H. 1977, ApJ, 217, 425

Pinilla, P., Birnstiel, T., Ricci, L., et al. 2012, A\&A, 538, A114

Pinte, C., Dent, W. R. F., Menard, F., et al. 2015, arXiv:1508.00584

Pollack, J. B., et al. 1994, ApJ, 421, 615

Regály, Z., Juhász, A., Sándor, Z., \& Dullemond, C. P. 2012, MNRAS, 419, 1701

Robitaille, T. P., Whitney, B. A., Indebetouw, R., \& Wood, K. 2007, ApJS, 169, 328

Ruge, J. P., Wolf, S., Uribe, A. L., \& Klahr, H. H. 2013, A\&A, 549, A97

Sargent, A. I., \& Beckwith, S. V. W. 1991, ApJ, 382, L31

Shen, Y., \& Turner, E. L. 2008, ApJ, 685, 553

Tamayo, D., Triaud, A. H. M. J., Menou, K., \& Rein, H. 2015, ApJ, 805, 100 
Testi, L., Birnstiel, T., Ricci, L., et al. 2014, Protostars and Planets VI, 339

Weingartner, J. C., \& Draine, B. T. 2001, ApJ, 548, 296

White, R. J., \& Hillenbrand, L. A. 2004, ApJ, 616, 998

Zhang, K., Blake, G. A., \& Bergin, E. A. 2015, ApJ, 806, L7

Zhang, X., Li, H., Li, S., \& Lin, D.N.C. 2014, ApJ, 789, L23

Zhu, Z., Stone, J.M., \& Bai, X. 2015, ApJ, 801, article id. 81

Zubko, V. G., et al. 1996, MNRAS, 282, 1321

This preprint was prepared with the AAS $\mathrm{LAT}_{\mathrm{E}} \mathrm{X}$ macros v5.2. 